\title{
Cherenkov radiation-based optical fibre diagnostics of fast electrons generated in intense laser-plasma interactions
}

\author{
H. Liu, G.-Q. Liao, Y.-H. Zhang, B.-J. Zhu, Z. Zhang, Y.-T. Li, \\ G. G. Scott, D. R. Rusby, C. Armstrong, E. Zemaityte, \\ D. C. Carroll , S. Astbury, P. Bradford, N. C. Woolsey, \\ P. McKenna and D. Neely
}

\section{Published version information}

Citation: H Liu et al. "Cherenkov radiation-based optical fibre diagnostics of fast electrons generated in intense laser-plasma interactions." Review of Scientific Instruments, vol. 89, no. 8 (2018): 083302.

DOI: $\underline{10.1063 / 1.5024872}$

This article may be downloaded for personal use only. Any other use requires prior permission of the author and AIP Publishing.

This version is made available in accordance with publisher policies. Please cite only the published version using the reference above. This is the citation assigned by the publisher at the time of issuing the APV. Please check the publisher's website for any updates. 


\section{Cherenkov radiation-based optical fibre diagnostics of fast electrons generated in intense laser-plasma interactions $\odot$}

Cite as: Rev. Sci. Instrum. 89, 083302 (2018); https://doi.org/10.1063/1.5024872

Submitted: 05 February 2018 . Accepted: 21 July 2018. Published Online: 08 August 2018

H. Liu, G.-Q. Liao, Y.-H. Zhang (D), B.-J. Zhu, Z. Zhang (D, Y.-T. Li (D, G. C. Scott, D. R. Rusby, C. Armstrong, E. Zemaityte, D. C. Carroll (D), S. Astbury, P. Bradford, N. C. Woolsey (D, P. McKenna (D), and D. Neely

\section{COLLECTIONS}

F This paper was selected as Featured

SC) This paper was selected as Scilight

\section{ARTICLES YOU MAY BE INTERESTED IN}

Time resolved detection of two-plasmon decay using three-halves harmonic emission on the National Ignition Facility

Review of Scientific Instruments 89, 083504 (2018); https://doi.org/10.1063/1.5038094

Novel scintillator-based $\mathrm{x}$-ray spectrometer for use on high repetition laser plasma interaction experiments

Review of Scientific Instruments 89, 073502 (2018); https://doi.org/10.1063/1.5019213

A study of picosecond laser-solid interactions up to $10^{19} \mathrm{~W} \mathrm{~cm}^{-2}$

Physics of Plasmas 4, 447 (1997); https://doi.org/10.1063/1.872103

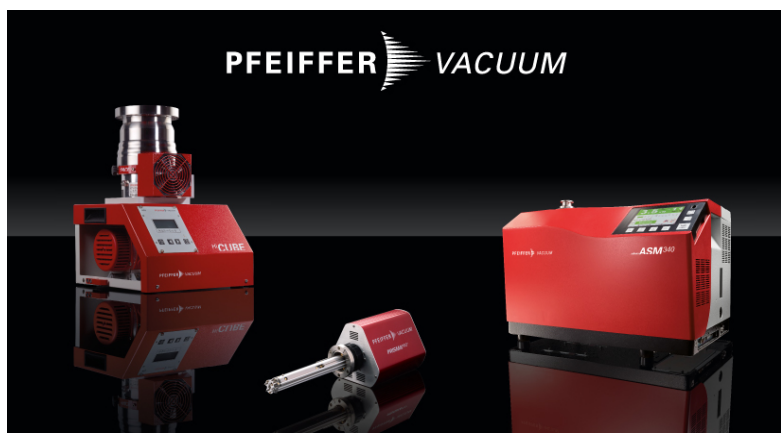

\section{Vacuum solutions from a single source}

Pfeiffer Vacuum stands for innovative and custom vacuum solutions worldwide, technological perfection, competent advice and reliable service.

Learn more! 


\title{
Cherenkov radiation-based optical fibre diagnostics of fast electrons generated in intense laser-plasma interactions
}

\author{
H. Liu, ${ }^{1,2}$ G.-Q. Liao, ${ }^{3}$ Y.-H. Zhang, ${ }^{1,2}$ B.-J. Zhu, ${ }^{1,2}$ Z. Zhang, ${ }^{1}$ Y.-T. Li, ${ }^{1,2,4, a)}$ G. G. Scott, ${ }^{5}$ \\ D. R. Rusby, ${ }^{5,6}$ C. Armstrong, ${ }^{5,6}$ E. Zemaityte, ${ }^{5,6}$ D. C. Carroll, ${ }^{5}$ S. Astbury, ${ }^{5}$ P. Bradford, ${ }^{7}$ \\ N. C. Woolsey, ${ }^{7}$ P. McKenna, ${ }^{6}$ and D. Neely $\left.5,6, a\right)$ \\ ${ }^{1}$ Beijing National Laboratory for Condensed Matter Physics, Institute of Physics, Chinese Academy of Sciences, \\ Beijing 100190, China \\ ${ }^{2}$ School of Physical Sciences, University of Chinese Academy of Sciences, Beijing 100049, China \\ ${ }^{3}$ Key Laboratory for Laser Plasmas (MoE) and School of Physics and Astronomy, Shanghai Jiao Tong University, \\ Shanghai 200240, China \\ ${ }^{4}$ Collaborative Innovation Center of IFSA (CICIFSA), Shanghai Jiao Tong University, Shanghai 200240, China \\ ${ }^{5}$ Central Laser Facility, Rutherford Appleton Laboratory, Didcot, Oxfordshire OX11 OQX, United Kingdom \\ ${ }^{6}$ Department of Physics, SUPA, University of Strathclyde, Glasgow G4 ONG, United Kingdom \\ ${ }^{7}$ Department of Physics, York Plasma Institute, University of York, Heslington York YO10 5DD, United Kingdom
}

(Received 5 February 2018; accepted 21 July 2018; published online 8 August 2018)

Diagnosing fast electrons is important to understand the physics underpinning intense laser-produced plasmas. Here, we demonstrate experimentally that a Cherenkov radiation-based optical fibre can serve as a reliable diagnostic to characterize the fast electrons escaping from solid targets irradiated by ultraintense laser pulses. Using optical fibre loops, the number and angular distributions of the escaping electrons are obtained. The data agree well with measurements made using image plate stacks. The optical fibre can be operated at high-repetition rates and is insensitive to x-rays and ion beams, which makes it advantageous over other routinely used fast electron diagnostics in some aspects. Published by AIP Publishing. https://doi.org/10.1063/1.5024872

\section{INTRODUCTION}

The study of fast electrons generated in interactions between a relativistically intense laser pulse $\left(>10^{18} \mathrm{~W} / \mathrm{cm}^{2}\right)$ and a solid target is important for understanding laser-plasma interaction physics and applications. ${ }^{1-7}$ Electrons at the target front surface are forward or backward accelerated with various mechanisms such as resonant absorption, ${ }^{8,9}$ vacuum heating, ${ }^{10}$ and $\mathrm{J} \times \mathrm{B}$ heating. ${ }^{11}$ When the forward electrons reach the target rear surface, a sheath field builds up, which reflects the electrons, impeding their escape. Diagnosing the total number, angular distribution, and energy spectra of the electrons which do escape contributes to understanding the efficiency and mechanisms of laser absorption ${ }^{12}$ and the electron transport in the target. ${ }^{7,13}$ The measurements also help us to characterize secondary processes such as the generation of high energy ions, ${ }^{14} \mathrm{X}$-rays, ${ }^{6}$ and $\mathrm{THz}$ radiation. ${ }^{15}$

High repetition rates are becoming more common for many of the new ultra-intense laser facilities currently being commissioned, which are capable of delivering high power laser pulses onto the target at a frequency of $1 \mathrm{~Hz}$ or faster. ${ }^{16,17}$ The image plate (IP) ${ }^{18}$ is widely used to detect escaping fast electrons. However, it can only be used at low repetition rates or in a single shot operation mode due to the unavoidable time required for the expense of reloading and scanning. Phosphors and scintillators are commonly used for high-repetition electron beam measurements. However, phosphors and

a) Authors to whom correspondence should be addressed: ytli@iphy.ac.cn and david.neely@stfc.ac.uk scintillators as well as IP are sensitive not only to electrons, but also to X-rays and ions, ${ }^{19,20}$ which are all generated when intense laser pulses irradiate solid targets. This can result in high background noise levels on these detectors. In addition, IP stacks or scintillator screens typically occupy a large solid angle, potentially making them incompatible with the simultaneous operation of other diagnostics.

Cherenkov radiation can be generated when an electron passes through an optical fibre at a speed greater than the local phase velocity of light. ${ }^{21}$ The technique is widely used to characterize the radiation dose in electron beam therapy. ${ }^{22}$ With a specially designed target, the energy distribution of fast electrons inside the target has been studied by measurement of the induced Cherenkov emission. ${ }^{23,24}$ In this paper, using a silica optical fibre based on Cherenkov radiation, we have measured the number and angular distributions of the escaping electrons in an ultra-intense laser-solid interaction experiment. We find that the signal intensity of Cherenkov photons inside the optical fibre depends linearly on the escaping electron number. Based on the linearity, a wraparound array of curved fibre loops is built to measure the angular distribution of the electrons. The results agree well with those measured using IP stacks.

\section{THEORY OF CHERENKOV RADIATION INDUCED BY ELECTRONS IN AN OPTICAL FIBRE}

When a non-monoenergetic electron beam passes through the silica core of a multimode step-index optical fibre, a number of Cherenkov photons are generated. After a propagation length of $L$, the total energy of the Cherenkov photons at a wavelength $\lambda$ remaining in the fibre can be written as $^{25}$ 


$$
\begin{aligned}
d E_{c} / d \lambda= & \pi^{2} h c D^{2} l /\left(274 \sin \psi_{e}\right) \lambda^{-3} T_{f i b r e}(L, \lambda) \\
& \times \int \eta f(E) \sin ^{2}\left(\theta_{c}\right) d E,
\end{aligned}
$$

where $h$ is Planck's constant, $c$ is the speed of light, $D$ is the core diameter, $l$ is the length of the fibre segment exposed to the electron beam, incidence angle $\psi_{e}$ is defined as the angle between the electron beam direction and fibre axis, $T_{\text {fibre }}(L, \lambda)$ is the fibre transmission, $f(E)$ is the flux of the electron beam incident on the fibre segment, $\eta$ is the collection efficiency which can be represented as

$$
\eta=1 / \pi * \arccos \left(\left(\sqrt{1-\sin ^{2}\left(\theta_{A}\right)}-\cos \psi_{e} \cos \theta_{c}\right) /\left(\sin \psi_{e} \sin \theta_{c}\right)\right),
$$

where $\theta_{c}=\arccos (1 /(n \beta)), n$ is the core refraction index, $\beta$ $=v_{e} / c$ is the speed of an electron in units of $c$, and $\theta_{A}=$ $\arcsin (N A / n)$, where NA is the numeric aperture of the fibre.

According to Eq. (1), the intensity of the excited Cherenkov signal is dependent on the parameters of the fibre and electron beam. The core diameter determines both the path length of an electron inside the optical fibre and the effective area exposed to the electron beam. A larger core diameter will generate more Cherenkov photons due to the $D^{2}$ dependence. Increasing the length of the fibre segment exposed to the electron beam also enhances the Cherenkov signal. For optical rays inside the fibre core, only the rays satisfying the condition that the angle between the propagation direction and fibre axis is smaller than the acceptance angle $\theta_{A}$ are collected. According to Eq. (2), to increase the number of Cherenkov photons collected, multimode fibres with a larger numeric aperture are preferred. Figure 1(a) shows that the incidence angle of the electrons plays a key role in the energy selection. When the incidence angle is set to be $20^{\circ}$, the fibre can only collect Cherenkov photons induced by electrons with energy between 190 and $450 \mathrm{keV}$. However, the fibre can collect those induced by high energy electrons $(\beta \sim 1)$ with a near-constant efficiency if the incidence angle is increased to $50^{\circ}$. The emitted Cherenkov light at a wavelength below $500 \mathrm{~nm}$ will experience significant absorption loss with the propagation length in high-OH and low-OH silica-core fibre, as shown in Fig. 1(b). It is obvious that high-OH fibres are advantageous in transporting Cherenkov photons because of their higher transmission in the violet and ultraviolet region. Therefore, to obtain a strong
Cherenkov signal induced by relativistic electrons, a high-OH, silica-core large NA multimode fibre with an incidence angle of $\sim 45^{\circ}$ is preferred.

The ultra-short Cherenkov light pulses induced by fast electrons will be broadened temporally when propagating inside an optical fibre. For silica-core step-index multimode fibres, pulse broadening effect mainly results from the intermodal dispersion. The initial Cherenkov light pulse duration is approximately equal to that of the escaping electron pulse, which is effectively negligible in comparison to modal dispersion effects. As a result, the rms pulse width $\sigma_{\text {out }}$ in the output of the fibre can be calculated as ${ }^{26}$

$$
\sigma_{\text {out }} \cong \sigma_{\text {mod }} \approx(N A)^{2} \cdot L /(4 \sqrt{3} c n),
$$

where $\sigma_{m o d}$ is the rms broadening of intermodal dispersion. To separate two Cherenkov light pulses induced at two different positions along the fibre, the minimum length of the fibre, $l_{\min }$, between the two points should satisfy

$$
l_{\min } \gtrsim(N A)^{2} * L /\left(4 \sqrt{3} n^{2}\right) .
$$

\section{EXPERIMENTS AND RESULTS}

\section{A. Waveform and spectrum of the Cherenkov light pulse in a single optical fibre}

The experiment is performed with the Vulcan laser facility at the Rutherford Appleton Laboratory (RAL), UK. Figure 2 shows the experimental setup. A $1053 \mathrm{~nm}$ laser pulse with a duration of $1 \mathrm{ps}$ and energies up to $400 \mathrm{~J}$ is focused onto

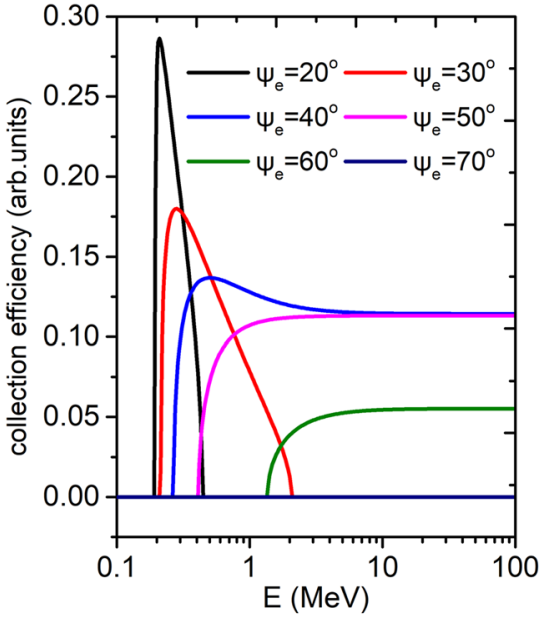

(a)

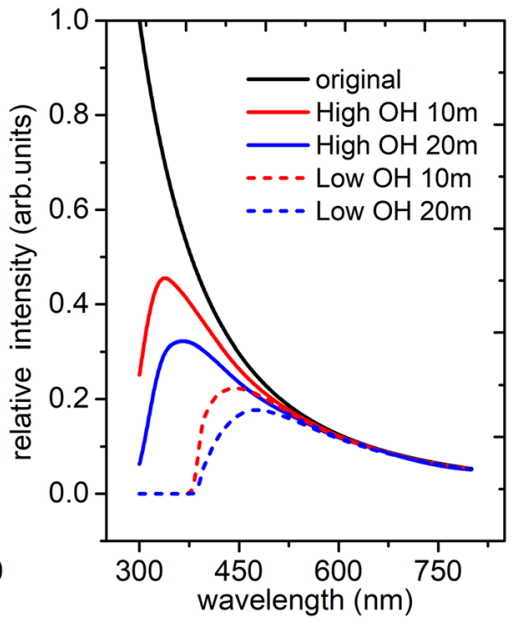

(b)
FIG. 1. (a) Collection efficiency as a function of electron energy with different incidence angles $\psi_{e}$ and numeric aperture $N A=0.39$. (b) Attenuated Cherenkov spectra from the high-OH and low-OH silica-core fibre under different transmission lengths. 


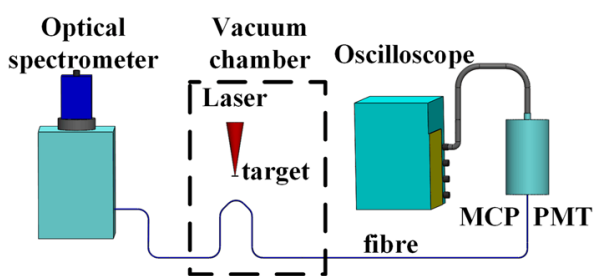

FIG. 2. Experimental schematic layout to measure the waveform and spectrum of the Cherenkov light induced by escaping electrons in an optical fibre.

a $100 \mu \mathrm{m}$ thick Au foil target, resulting in a peak intensity of $2 \times 10^{20} \mathrm{~W} / \mathrm{cm}^{2}$ with a focal spot of $5 \mu \mathrm{m}$ (FWHM). A $200 \mu \mathrm{m}$ silica-core multimode step-index fibre is placed behind the foil target to sample the fast electrons escaping from the target rear. The induced photons propagate along the fibre in two opposite directions and exit at both ends of the fibre. One end is coupled into a MCP photomultiplier (Photek model PMT210) with a $\mathrm{MgF}_{2}$ input window, whose time resolution can reach $150 \mathrm{ps}$ and gain approximate $10^{6}$ with $4.7 \mathrm{kV}$ applied. The amplified light signal is recorded on a $13 \mathrm{GHz}$-bandwidth oscilloscope. The optimum sensitivity of the photomultiplier tube (PMT) is between $300 \mathrm{~nm}$ and $500 \mathrm{~nm}$ and decreases rapidly for longer wavelengths. ${ }^{27}$ The other end is connected to an optical spectrometer (Andor Shamrock SR-303i) to measure the spectrum of the Cherenkov light.

The number of Cherenkov photons generated in the core is about 1.8 times of that in the cladding with the diameter ratio between them of 1.25 . Moreover, light spreading in the cladding experiences much higher power loss than that in the core. Therefore, the majority of the signal is from the silica core of the fibre. Figure 3(a) shows a typical signal from the PMT, which suffers little electromagnetic noise. ${ }^{28}$ The temporal width of the signal is $0.8 \mathrm{~ns}$ (FWHM). The expected pulse broadening due to intermodal dispersion is around $0.78 \mathrm{~ns}$ after a propagation length of $15.5 \mathrm{~m}$ in the fibre. Factoring in the time resolution of the PMT, the original pulse width is estimated at a level of several picoseconds. This is much different from the typical light pulse generated by the scintillation process, which has a short rise time and long decay time with duration more than several nanoseconds. ${ }^{29}$ Considering the pulse duration and waveform, the instantaneous Cherenkov radiation is one of the most possible processes accounting for the measured nanosecond light pulses. Figure 3(b) shows a typical Cherenkov light spectrum measured by the optical spectrometer. The spectrum is continuous and broad from $400 \mathrm{~nm}$ to $800 \mathrm{~nm}$ rather than a peaked spectrum which is a signature of most scintillation processes. The measured spectrum is well reproduced by the theoretical one after considering the spectral attenuation of the fibre and sensitivity of the spectrometer, as shown in Fig. 3(c). This provides additional evidence of the measured light coming from the Cherenkov radiation.

Escaping electrons, high energy protons, and X-rays are simultaneously generated in our experiment. The energy threshold of electrons that can induce Cherenkov radiation in the silica optical fibre is only $190 \mathrm{keV}$, which is much lower than the electron temperature $\sim \mathrm{MeV}$ in our experiment. The threshold is $1.3 \mathrm{GeV}$ for protons. In our experiment, the maximum proton energy is about $30 \mathrm{MeV}$, far from the threshold triggering the Cherenkov radiation. In intense laser-solid interaction experiments, another main emission is X-rays from hundreds of $\mathrm{eV}$ to tens of keV. However, X-ray with such energy cannot induce Cherenkov radiation. In addition, gamma rays could also induce Cherenkov radiation by electron-positron pair production in an optical fibre. However, this contribution is negligible compared with that induced by the relativistic escaping electrons for thin, low to mid Z solid targets. Therefore, the measured Cherenkov emission in the fibre in our experiment is mainly induced by the escaping electrons.

\section{B. A fibre loop as an escaping electron number monitor}

A curved fibre array is designed to measure the escaping electron number with the Cherenkov radiation. Figure 4 shows the experimental layout and a picture of the array. The array consists of 8 curved fibre loops wounded from a single
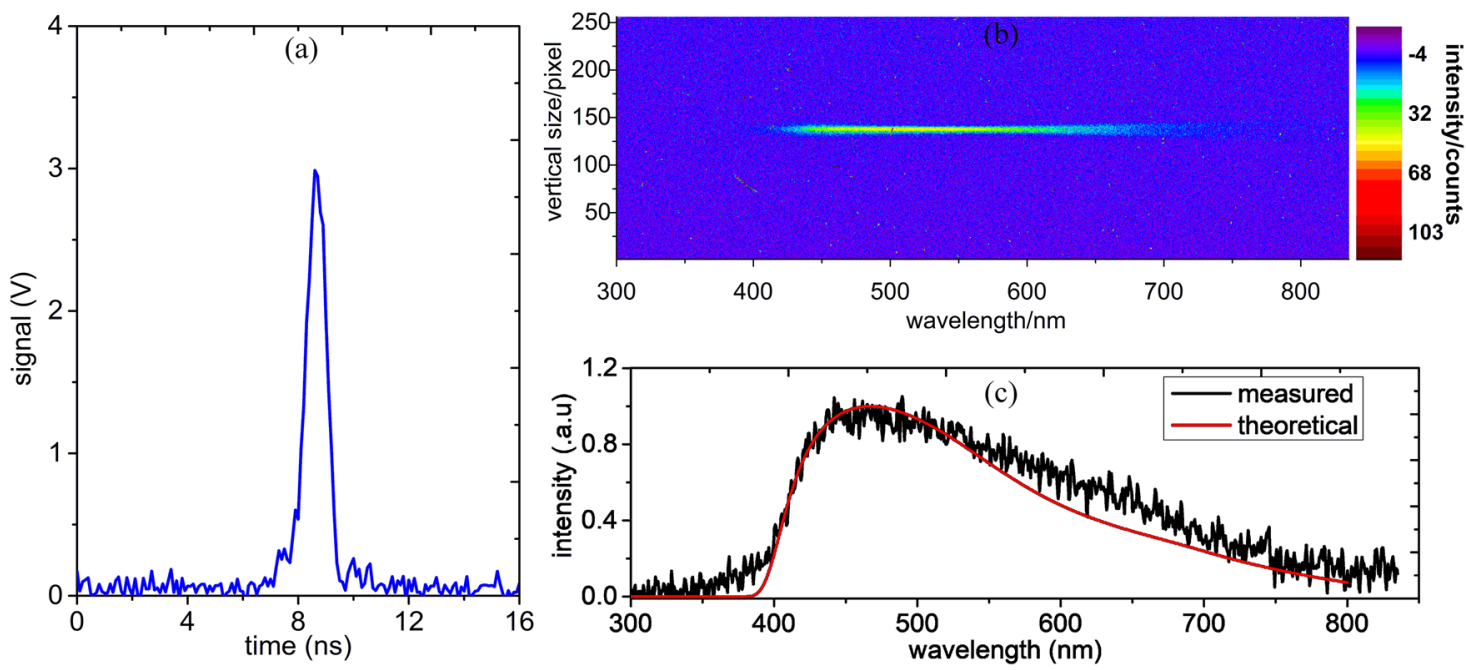

FIG. 3. (a) Typical Cherenkov signal from a $200 \mu \mathrm{m}$ diameter silica-core multimode step-index fibre after propagating $15.5 \mathrm{~m}$ measured by the PMT. (b) Spectrum of Cherenkov light after propagating $10 \mathrm{~m}$ measured by the optical spectrometer. (c) Measured versus theoretical Cherenkov spectrum. 


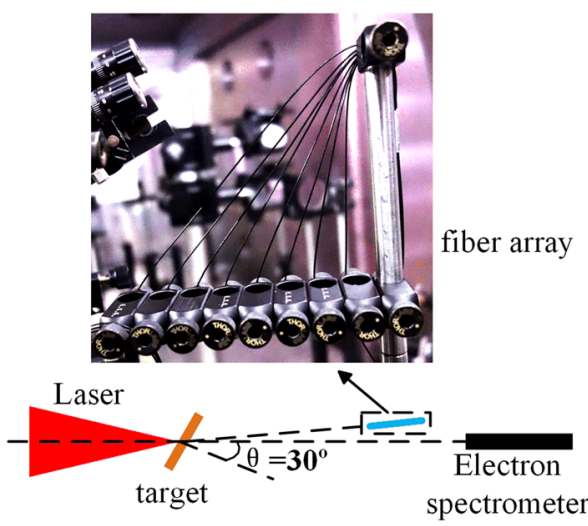

FIG. 4. Experimental layout of the fibre array consisting of 8 curved loops and an electron spectrometer.

long fibre. The shapes of the loops are right-angle triangles with different hypotenuse angles from $41^{\circ}$ to $82^{\circ}$. The horizontal sections of the fibre along the right-angle side near the laser propagation direction are shielded from the escaping electrons by $12 \mathrm{~mm}$ diameter stainless steel posts. The Cherenkov photons generated in the vertical fibre sections along the other right-angle side cannot be collected due to the large incident angle. As a result, only the fibre sections along the hypotenuses produce Cherenkov signals of the escaping electrons. The output Cherenkov signals from one end of the loops are directed into a MCP-PMT, similar to that in Fig. 2.

We use an electron spectrometer with a $0.2 \mathrm{~T}$ magnet and a calibrated image plate detector to calibrate the curved fibre array. The spectrometer is installed in the laser propagation direction to measure the electrons from the rear target surface. The loop array is set up beside the electron spectrometer with the angle between them smaller than $2^{\circ}$.

The calculated electron energy-spectral sensitivity for each fibre loop is shown in Fig. 5(a). Each loop can only respond to the electrons above a specific energy, which is determined by the hypotenuse angles. The minimum energies are $200 \mathrm{keV}$ for loop 1 and $2.5 \mathrm{MeV}$ for loop 8, respectively.
According to Fig. 5(a), all the loops have a relatively flat spectral response of for high energy electrons. This means that the number of high energy electrons can be directly estimated based on the signal intensity without any assumption for the energy distribution.

Figure 5(b) shows a typical Cherenkov light signal measured by the fibre array, taken from a $100 \mu \mathrm{m}$ copper foil target driven by a $100 \mathrm{~J}, 2$ ps laser pulse at an intensity of $10^{20} \mathrm{~W} / \mathrm{cm}^{2}$. There are 8 light pulses presented, which correspond to the 8 loops. The gaps between the pulses are due to the nil response of the fibre sections along the two right-angle sides. A five-turn fibre coil is set in the plane vertical to the laser propagation direction, with the same distance and height from the target as the right-triangle fibre loops. The measured signal from the fibre coil is negligible compared to the signal from the fibre loops, which further proves the incidence angle dependence of Cherenkov radiation.

A typical energy spectrum of the escaping electrons measured by the electron spectrometer is shown in Fig. 5(c). The spectrum is fitted with a Maxwell distribution with an electron temperature of $2.5 \mathrm{MeV}$. It is found that the temperature is in the range of 2-3 MeV when the laser parameters are changed. However, the electron number varies considerably. The number of the escaping electrons is calculated using the measured spectrum by integrating the number of electrons between $1 \mathrm{MeV}$ and $10 \mathrm{MeV}$. Considering the relative sensitivity of the eight loops, the pulse signal from loop 3 is integrated over time to characterize the electron number. The comparison between them is shown in Fig. 5(d), based on the data obtained from 20 shots in the experiment. A linear correlation between the number of electrons and integrated intensity of the pulsed Cherenkov signal is found.

\section{Wraparound array of fibre loops as an angular distribution diagnostic of escaping electrons}

Eighteen fibre loops are wound using a single long fibre to measure the angular distribution of the escaping electrons.
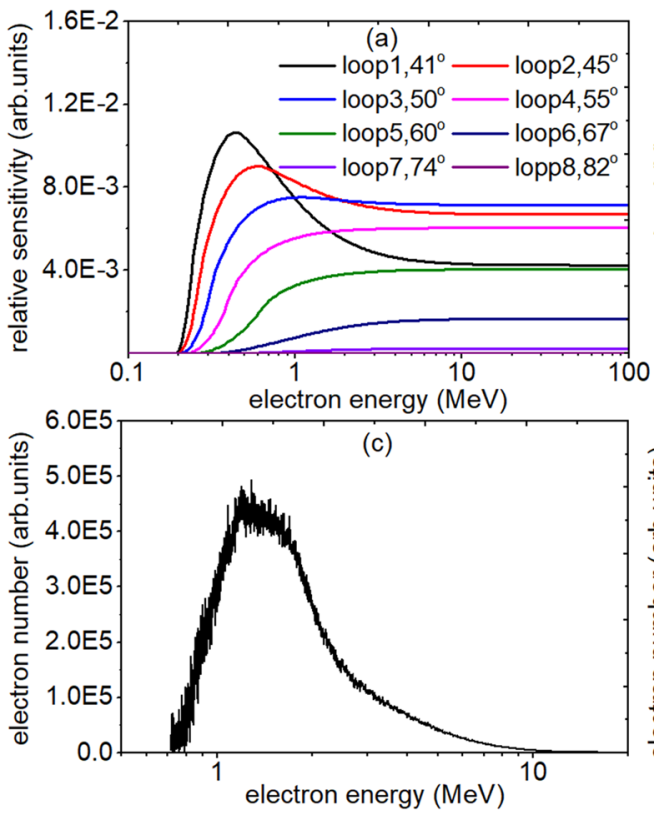
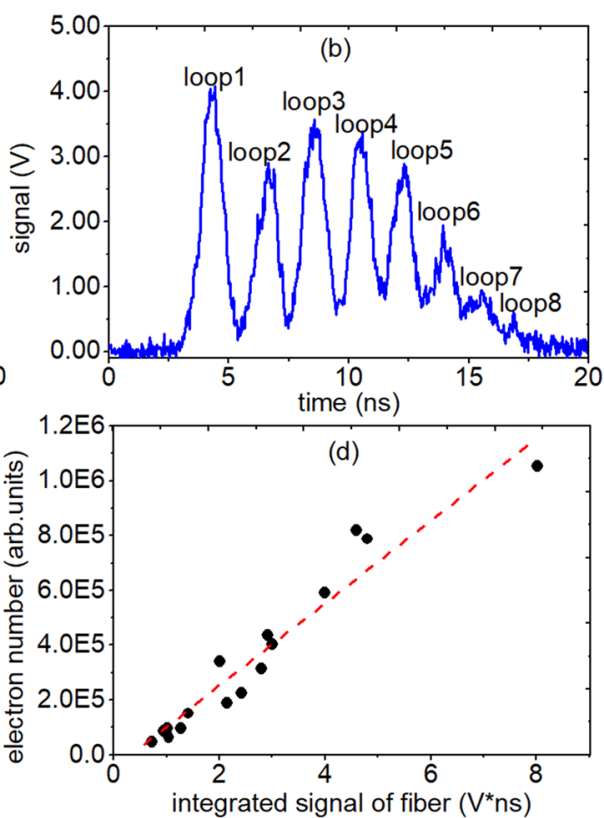

FIG. 5. (a) Electron energy dependent sensitivity of the 8 fibre loops. (b) Typical signal waveform of the fibre loops. (c) Typical electron energy spectrum measured by the electron spectrometer. (d) Correlation between the integrated pulse signal of loop 3 and the electron number between 1 and $10 \mathrm{MeV}$ measured by the electron spectrometer. 
(a)
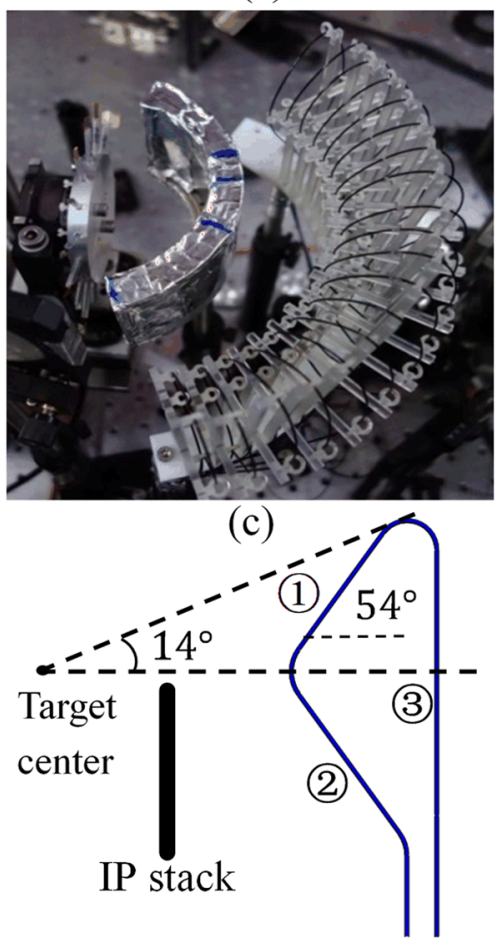

(b)

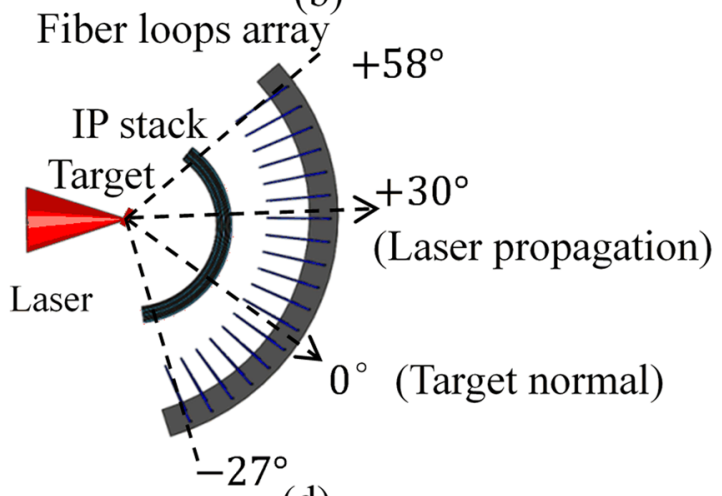

(d)

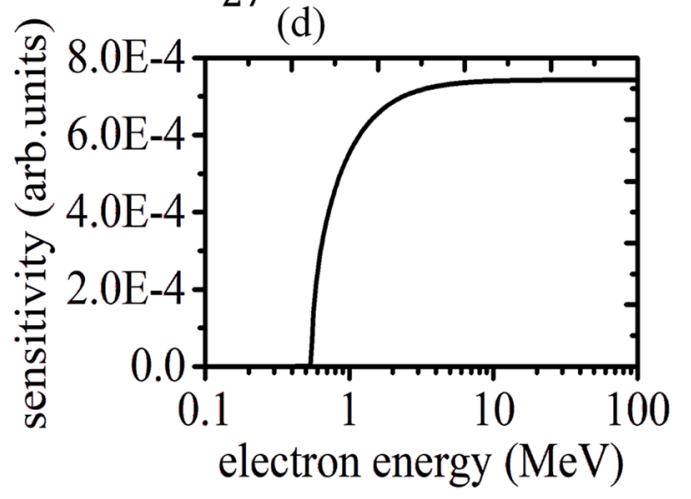

FIG. 6. (a) Photograph of the experimental layout of angular distribution measurement using IP stacks and a fibre loop array. The distances from the target center to IP stacks and the fibre loop array are $55 \mathrm{~mm}$ and $110 \mathrm{~mm}$, respectively. (b) Schematic top view of the layout in (a). (c) Schematic diagram of the setup of the curved fibre loop. (d) Energy dependent sensitivity of the curved fibre loop in (c).
As shown in Figs. 6(a) and 6(b), those fibre loops form a wraparound array centered around the target, which covers a horizontal angle of $85^{\circ}$ in total. The angular spacing between the adjacent loops is $5^{\circ}$. Figure 6(c) shows the geometrical structure of each loop, which is made up of three segments. Segment (1) is set for sampling the upper half of the escaping beam. Segment (2) is for the lower half originally, but it is blocked by the IP stacks in the experiment. The incident angle of the electrons onto the segment (1) is between $40^{\circ}$ and $54^{\circ}$. Figure 6(d) shows the sensitivity of segment (1), which mainly respond to relativistic electrons. The incidence angle onto the segment (3) is so large that Cerenkov photons generated in it cannot be collected. As a result, segment (3) and segment (2) generate a time separation between the Cherenkov signals from two adjacent loops.
Wraparound IP stacks ${ }^{7}$ are installed to calibrate the relative sensitivity of the fibre loop array. The stacks are situated in the lower half of the escaping electron beam covering an angular range to the target normal from $-35^{\circ}$ to $+75^{\circ}$.

A $100 \mu \mathrm{m}$ copper foil target was shot with the laser beam at the best focus to calibrate the relative sensitivity of the fibre loops. Figure 7(a) shows the angular distribution of the escaping electrons measured on the first layer of the IP stacks, which is mainly sensitive to electrons more than $2 \mathrm{MeV}^{7}$ There are two electron beams observed. The main beam is in the target normal direction, and the other (weaker) one is in the laser propagation direction. Figure 7(b) shows the signal from the fibre loop array, consisting of eighteen fully separated pulses. Due to the intermodal dispersion, the signals are broadened increasingly as the Cherenkov light (a)

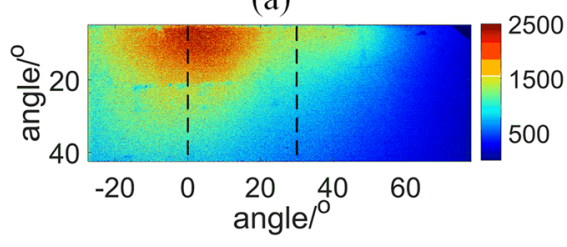

(c)

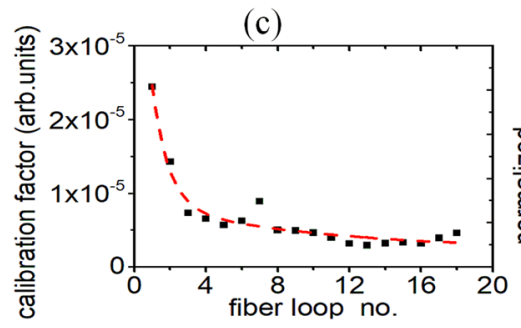

(b)

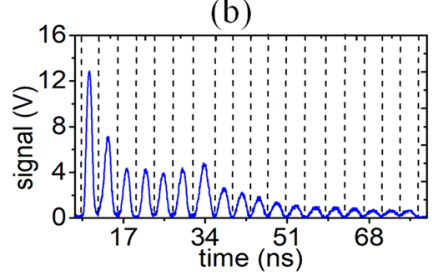

(d)

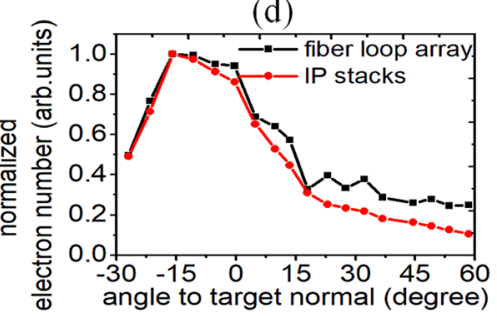

FIG. 7. (a) Angular distribution measured by the wraparound IP stacks. The black dashed lines at $0^{\circ}$ and $30^{\circ}$ represent the target normal and laser propagation direction, respectively. (b) Cherenkov signal from the array of the fibre loops. (c) Calibration factors of the fibre loop array obtained by comparing with the IP measurement. (d) Comparison of the angular distributions measured by the calibrated fibre loop array and wraparound IP stacks. 
travels through a greater number of loops before reaching the detector.

The electron number incident onto each fibre loop is estimated by integrating the signal on the IP over the areas corresponding to each loop. The calibration factor of each loop is calculated as the ratio of the Cherenkov signal intensity and the estimated electron number from the IP, as shown in Fig. 7(c). For the first three loops, the exponential decrease of the calibration factor is due to the huge bend $\operatorname{loss}^{30}$ in the fibre. The calibration factors of the last 10 loops are near-constant because the bend loss becomes saturated with the multi-turn layout. ${ }^{31}$ This bend loss saturation is due to the near complete rejection of the high modes after a certain length of the bent fibre. However, the low-order modes can still remain to propagate inside the core with a negligible bend loss.

With the calibration factors, the angular distribution of the escaping electrons can be calculated using the Cherenkov signals from the fibre loop array. To verify the validity of this diagnosing method, we took another laser shot with the IP stacks and the fibre loop array unchanged. Good agreement is found between the angular distribution of escaping electrons given by the calibrated fibre loop array and the IP stacks as shown in Fig. 7(d).

\section{CONCLUSION}

Cherenkov radiation induced by escaping electrons inside a silica-core multimode optical fibre has been characterized with a series of measurements carried out in intense laserplasma interaction experiments. The waveform and spectrum of Cherenkov light pulses in a single optical fibre are measured. A linear correlation is observed between the intensity of the Cherenkov light from a fibre loop and the escaping electron number. A calibrated fibre loop array is designed to measure the angular distribution of escaping electrons, in good agreement with that measured by IP stacks. Apart from the angular distribution, the energy spectral characteristic of escaping electrons can also be measured potentially with different incidence angles set for an optical fibre. Compared with other conventional diagnostics of fast electrons, the Cherenkov radiation-based optical fibre shows advantages, including the potential to operate at high repetition, low ion and X-ray sensitivity, and flexible size and shape, making it suitable for next-generation high-repetition laser-plasma experiments.

\section{ACKNOWLEDGMENTS}

This work is supported by the Newton China-UK joint research grant on laser-ion acceleration and novel Terahertz radiation, and EPSRC (Grant Nos. EP/K022415/1 and EP/R006202/1). This work is also supported by the National Basic Research Program of China (Grant No. 2013CBA01501), the National Nature Science Foundation of China (Grant Nos. 11520101003 and 11535001), and the Strategic Priority Research Program of the Chinese Academy of Sciences (Grant Nos. XDB16010200 and XDB07030300). G.Q.L. would like to acknowledge support from the National Postdoctoral Program for Innovative Talents (Grant No. BX201600106).

Data associated with research published in this paper can be accessed at: https://doi.org/10.5286/edata/711.

${ }^{1}$ M. H. Key et al., Phys. Plasmas 5, 1966 (1998).

${ }^{2}$ P. Gibbon and E. Forster, Plasma Phys. Controlled Fusion 38, 769 (1996).

${ }^{3}$ J. J. Honrubia and J. Meyer-Ter-Vehn, Plasma Phys. Controlled Fusion 51, 014008 (2009).

${ }^{4}$ A. R. Bell and R. J. Kingham, Phys. Rev. Lett. 91, 035003 (2003).

${ }^{5}$ Y.-T. Li, X. H. Yuan, M. H. Xu et al., Phys. Rev. Lett. 96, 165003 (2006).

${ }^{6}$ Z.-M. Sheng, Y. Sentoku, K. Mima et al., Phys. Rev. Lett. 85, 5340 (2000).

${ }^{7}$ D. R. Rusby, L. A. Wilson, R. J. Gray et al., J. Plasma Phys. 81, 475810505 (2015).

${ }^{8}$ P. Gibbon and A. R. Bell, Phys. Rev. Lett. 68, 1535 (1992).

${ }^{9}$ S. C. Wilks and W. L. Kruer, IEEE J. Quantum Electron. 33, 1954 (1997).

${ }^{10}$ F. Brunel, Phys. Rev. Lett. 59, 52 (1987).

${ }^{11}$ S. C. Wilks, W. L. Kruer, M. Tabak, and A. B. Langdon, Phys. Rev. Lett. 69, 1383 (1992).

${ }^{12}$ D. F. Cai, Y. Q. Gu, Z. J. Zheng et al., Phys. Plasmas 10, 3265 (2003).

${ }^{13}$ M. N. Quinn, X. H. Yuan, X. X. Lin et al., Plasma Phys. Controlled Fusion 53, 025007 (2011).

${ }^{14}$ S. C. Wilks, A. B. Langdon, T. E. Cowan et al., Phys. Plasmas 8, 542 (2001).

${ }^{15}$ G. Q. Liao, Y. T. Li, Y. H. Zhang et al., Phys. Rev. Lett. 116, 205003 (2016).

${ }^{16}$ J. S. Green, M. Borghesi, C. M. Brenner et al., Proc. SPIE 8079, 807919 (2011).

${ }^{17}$ C. Danson, D. Hillier, N. Hopps et al., High Power Laser Sci. Eng. 3, e3 (2015).

${ }^{18}$ I. J. Paterson, R. J. Clarke, N. C. Woolsey et al., Meas. Sci. Technol. 19, 095301 (2008).

${ }^{19}$ Y. Glinec, J. Faure, A. Guemnietafo et al., Rev. Sci. Instrum. 77, 103301 (2006).

${ }^{20}$ G. C. Tyrrell, Nucl. Instrum. Methods Phys. Res., Sect. A 546, 180 (2005).

${ }^{21}$ K. Tanaka, Phys. Rev. 93, 459 (1954).

${ }^{22}$ B. Lee, K. W. Jang, H. C. Dong et al., Nucl. Instrum. Methods Phys. Res., Sect. A 579, 344 (2007).

${ }^{23}$ F. Brandl, G. Pretzler, D. Habs et al., Europhys. Lett. 61, 632 (2003).

${ }^{24}$ H. Habara, K. Ohta, K. A. Tanaka et al., Phys. Rev. Lett. 104, 055001 (2010).

${ }^{25}$ Y. C. Wang, Y. W. Shi, and H. T. Jiang, Proc. SPIE 1572 (1991).

${ }^{26} \mathrm{G}$. Hill, The Cable and Telecommunications Professionals' Reference, $3 \mathrm{rd}$ ed. (Focal Press, 2008)

${ }^{27}$ See www.photek.com/products/photomultipliers.html/ for PMT210.

${ }^{28}$ M. J. Mead et al., Rev. Sci. Instrum. 75, 4225 (2004).

${ }^{29}$ L. Swiderski, M. Moszynski, A. Syntfeldkazuch et al., Nucl. Instrum. Methods Phys. Res., Sect. A 749, 68 (2014).

${ }^{30}$ D. Marcuse, J. Opt. Soc. Am. 66, 216 (1976).

${ }^{31}$ S. Moon and Z. Chen, Appl. Opt. 51, 8262 (2012). 